A NEW TOOL FOR BILATERAL DIAPHRAGMATIC PARALYSIS DIAGNOSIS

doi:10.1136/archdischild-2012-302724.0420

1JM Liet, 'F Barrière, 'B Gaillard-Le Roux, 'JM Dejode, 'N Joram, ${ }^{2} Y$ Péréon, ${ }^{3} \mathrm{~V}$ Gournay. 'Pediatric Intensive Care Unit, Hopital Mère-Enfant CHU of Nantes; 2Service d'Explorations Fonctionnelles, Hotel Dieu, CHU of Nantes; ${ }^{3} U n i t e ́$ de Pédiatrie Spécialisée, Hopital Mère-Enfant CHU of Nantes, Nantes, France

Background and Aims Bilateral diaphragmatic paralysis (BPD) is a rare cause of unexplained respiratory failure. Although it is a known complication of cardiothoracic surgery, it is often underrecognized and diagnosis is frequently delayed (Billings 2008). We report two children in whom BDP was easily detected using an esophageal probe equipped with sensors for measurement of electrical activity of the diaphragm.

Results Case 1: A 3-year-old boy with complex congenital cardiopathy underwent a third surgery for the bidirectional Glenn anastomosis procedure. Extubated few hours after surgery, he developed dyspnea. After reintubation, an esophageal probe equipped with sensors was installed. No electrical activity of the diaphragm could be found, thus evoking the diagnosis of BDP. This diagnosis was confirmed later by a fluoroscopy.

Case 2: A 9-month-old girl with atrioventricular canal defect underwent a third surgery for a mitral valve placement. Because of several extubation failures, tracheal fibroscopy, chest tomodensitometry, and an echography of the diaphragm performed by a radiologist did not provide an explanatory diagnosis. Thereafter an esophageal probe equipped with sensors did found electrical activity of the diaphragm, in the absence of blood alkalosis nor profound sedation. The diagnosis of BDP was confirmed by an electromyography of the diaphragm with a phrenic-nerve conduction study.

Conclusions Commercially available feeding tubes equipped with sensors permit to record electrical activity of the diaphragm via a ventilator using a standardized method (Sinderby 1997). This measurement allows a rapid diagnosis of bilateral diaphragmatic paralysis at the bedside.

\section{SYSTEMATIC ECHOCARDIOGRAPHY IN CHILDREN WITH ACUTE RESPIRATORY FAILURE (ARF) IN PICU OF THE UNIVERSITY HOSPITAL CENTER OF ORAN (ALGERIA)}

doi:10.1136/archdischild-2012-302724.0421

K El Halimi, H Bouguetof, MA Negadi, D Boumendil, ZC Mentouri. Pediatric Intensive Care Unit, Faculty of Medicine - Oran University, Oran, Algeria

Background and Aims Sytematic echocardiography in children with ARF in bedside allows the description of cardiac anatomy with the segmental analysis. The aim of this study is to determine the heart malformation as etiology of ARF.

Methods In this prospective study, 53 children with ARF had an echocardiography exploration from september 2009 to march 2012. Results 53 transthoracic echocardiography (TTE) were performed and congenital heart diseases were found in 29 patients.

Segmental analysis allow morphological and functional study of the heart; search for congenital defects and look for possible hémodynamic causes of ARF like high arterial pulmonary blood pressure.

Conclusion TTE is a non invasive tool useful to bedside of children in PICU for the dignostic of cardiac etiology of ARF. In developing countries systematic TTE must be performed to screening of congenital heart malformations.
M Rascanin. Neonatology, GAK 'Narodni Front', Belgrade, Serbia, Ruzica Djordjevic, OGC "Narodni front", Belgrade, Serbia

Introduction Surfactant is mix of lypo-proteins and it synthesizes in the lungs of the fetus. It lines the walls of the alveoli preventing their collapse and prevents athelectasis. The most important indication for use of surfactant is Respiratory Distress Syndrome (RDS) of the preterm infants.

Goal The goal of our paper is to represent our experience in application of surfactant immediately after birth in the prophylaxis of RDS in premature babies.

Method Retrospective analysis of neonatal morbidity of 92 infants during period 2010-2011, to whom prophylactic surfactant administered in our Neonatal Department. We assessed the b vitality at birth, body weight of newborns, gestational age and gender.

Results Analysis of gestational maturity was noted that the 4, $34 \%$ of infants had a gestational age of less than 25 weeks of gestation, $45,65 \%$ from 25,1 to 28 w., $21.19 \%$ from 28.1 to 30 and $22.82 \% \geq 30.1 \mathrm{w}$. The average gestational age was $28.3 \pm 1.8$ weeks. The average Apgar score in the fifth minute was 5.27 \pm 1.30 . The average body weight was $1086.85 \mathrm{~g} \pm 253,47 \mathrm{~g} .48 .92 \%$ were male and $51.08 \%$ was female. After surfactant therapy, they were transported to the tertiary level institutions in good general condition.

Conclusion Surfactant needs to be applied as early as possible and not allow the infant exhausted. Presence team of neonatologists and pediatric nurses will increase the percentage of infants who have the ability to lower the gestational maturity to be able to live.

\section{ACUTE RESPIRATORY FAILURE IN CHILDREN - A 3 YEARS EPIDEMIOLOGICAL STUDY}

doi:10.1136/archdischild-2012-302724.0423

D Pacurar, R Vlad, I Tincu, I Andronie, AM Pitran, R Smadeanu, C Zapucioiu, D Oraseanu. 'Grigore Alexandrescu' Children Emergency Hospital, Bucharest, Romania

Background and Aims Acute respiratory failure is the most frequent reason for admission in a pediatric emergency department. This study aimed to investigate the epidemiological characteristics of patients admitted with acute respiratory failure.

Methods We performed a retrospective study regarding the period September 2009-January 2012 that included all patients admitted with the diagnosis of acute respiratory failure in "Grigore Alexandrescu" Children Emergency Hospital in Bucharest; we evaluated personal data (sex, age), time of admission (year, month, day, hour, hospitalization period), admission diagnosis and co morbidities. Statistical analyze was performed with Microsoft Excel and SPSS.

Results We had 836 patients admitted for acute respiratory failure being $3.14 \%$ of all hospitalised patients and $5.4 \%$ of all patients suffering of respiratory diseases; the mean age of our study group was 2 years 9 months. Sex distribution indicates 537 boys and 299 girl, $\mathrm{p}=0.00$. The maximum incidence was in 2010 (mean 33cases/ month), in October (15.2\%), between 18.00-21.00 p.m.(17.6\%). In the majority of cases we claim that acute pneumonia (48.7\%) was the main cause of respiratory impairment followed by bronchiolitis (25.4\%) and asthma (9.1\%). Rare causes of respiratory failure were: intoxications, neurological conditions, cardiac malformations and diabetes.

Conclusions Acute respiratory failure still remains an important issue of pediatric emergency departments. The diseases complicated with acute respiratory failure are various and sometimes surprising. DIAGNOSIS AND TREATMENT IN CRITICALLY ILL CHILDREN 